\title{
Modeling few-body resonances in finite volume
}

\author{
Peng Guo® $\oplus^{1,2, *}$ \\ ${ }^{1}$ Department of Physics and Engineering, California State University, Bakersfield, California 93311, USA \\ ${ }^{2}$ Kavli Institute for Theoretical Physics, University of California, Santa Barbara, California 93106, USA
}

(Received 28 July 2020; accepted 8 September 2020; published 25 September 2020)

\begin{abstract}
Under the assumption of separable interactions, we illustrate how the few-body quantization condition may be formulated in terms of phase shifts, in general, which may be useful for describing and modeling few-body resonances in finite volume.
\end{abstract}

DOI: 10.1103/PhysRevD.102.054514

\section{INTRODUCTION}

Few-hadron dynamics plays an important role in hadron and nuclear physics. There have been many good examples of physics processes that can only be understood through few-body interactions, such as the $u$ - and $d$-quark mass difference in $\eta \rightarrow 3 \pi$ [1-7], Efimov states [8,9], and halo nuclei $[10,11]$. The understanding of few-body interactions is also crucial in recent experimental efforts in the study of exotic hadrons since most exotic hadron states are expected to appear as few-hadron resonances. On the theory side, lattice quantum chromodynamics (LQCD) provides an $a b$ initio method for the study of exotic hadron states. However, LQCD computation is usually performed in Euclidean space with certain periodic boundary conditions; normally, only discrete energy spectra are measured in numerical simulations. Hence, mapping out few-hadron dynamics from the discrete energy spectrum is a key step for the study of exotic hadron states in LQCD. In the two-body sector, the Lüscher formula [12] and its variants [13-22] provide an elegant form of mapping out two-body phase shifts from discrete energy levels.

In the past few years, much progress has been made using different approaches [23-54], going beyond the three-body threshold. Although few-body quantization conditions are formulated differently among these groups, it has been very clear [52] that in few-body sectors, the fewbody amplitudes are not directly extracted from lattice results. Particle interactions or their associated subprocess amplitudes are in fact essential ingredients in quantization conditions. The infinite volume few-body amplitudes that are generated by particle interactions through coupled

\footnotetext{
*pguo@csub.edu
}

Published by the American Physical Society under the terms of the Creative Commons Attribution 4.0 International license. Further distribution of this work must maintain attribution to the author(s) and the published article's title, journal citation, and DOI. Funded by SCOAP. integral equations must be computed in a separate step once these dynamical ingredients are determined. In order to make predictions or fit lattice results, dynamical ingredients of quantization conditions, such as interaction potentials or off-shell subprocess amplitudes, must be modeled one way or another. In addition, the number of partial waves involved in some physical processes may be large, which may add some extra complications on top of the uncertainty in modeling itself. Therefore, to have reliable and controllable predictions, the modeling of dynamical ingredients must be constrained or guided by experimental data or effective theory. Nevertheless, there are two physical regions in which predictions and calculations may be fairly reliable: (1) near threshold, which is the region where the physical reaction can be described rather precisely by nonrelativistic potential theory or relativistic effective perturbation theory; and (2) near resonances region, where resonance properties may be less affected by modeling and other partial waves.

In the present work, we focus on the near-resonance region and aim to provide an approximate means for the modeling of few-body resonances in finite volume. Based on the separable interaction potential assumption, we illustrate how the few-body quantization condition may be formulated in terms of subprocess phase shifts. Hence, the resonances may be modeled and inserted into the quantization condition through phase shifts. Both two-body and three-body subprocess amplitudes appear to be Lüscher formulalike, and solutions are given by algebra equations. The aim of this work is to illustrate a simple way to parametrize dynamics of few-body resonances in finite volume. Under the separable short-range potential approximation, the few-body formalism is greatly simplified, with the trade-off that the approximation may only be valid for the description of sharp resonance dynamics. As a simple illustration, the formalism is only presented in nonrelativistic kinematics; the extension to relativistic kinematics may be possible by replacing nonrelativistic few-body propagators with relativistic ones; 
see $[49,51]$. The relativistic extension of the formalism will be presented in future publications.

The paper is organized as follows. With a separable interactions approximation, the technical details of formulating quantization conditions in terms of phase shifts are presented in Sec. II. A summary is given in Sec. III.

\section{QUANTIZATION CONDITION UNDER THE SEPARABLE INTERACTIONS ASSUMPTION}

The few-body quantization condition in finite volume can be formulated from homogeneous Faddeev-type equations; see [50-53]. As a simple example, we consider three nonrelativistic identical bosons of mass $m$ interacting with both a pairwise interaction and a three-body force in what follows. Because of exchange symmetry, only two independent Faddeev amplitudes are required: $\mathcal{T}^{(2 b)}$ and $\mathcal{T}^{(3 b)}$, which are associated with the pairwise two-body interaction $V^{(2 b)}$ and the three-body interaction $V^{(3 b)}$ by

$$
\mathcal{T}^{(2 b, 3 b)}\left(\mathbf{k}_{1}, \mathbf{k}_{2}\right)=-\left\langle\mathbf{k}_{1} \mathbf{k}_{2}\left|m V^{(2 b, 3 b)}\right| \Psi\right\rangle,
$$

where $\Psi$ stands for the three-body total wave function. The $\left(\mathbf{k}_{1}, \mathbf{k}_{2}\right) \in \frac{2 \pi \mathbf{n}}{L}, \mathbf{n} \in \mathbb{Z}^{3}$ refer to particle-1 and -2 momenta, respectively, and the third particle momentum is constrained by total momentum conservation,

$$
\mathbf{k}_{3}=-\mathbf{k}_{1}-\mathbf{k}_{2} \text {. }
$$

In what follows, we also use the symbols $\left(\mathbf{k}_{13}, \mathbf{k}_{(13) 2}\right)$ to describe two independent relative momenta of three particles, where

$$
\begin{aligned}
\mathbf{k}_{13} & =\frac{\mathbf{k}_{1}-\mathbf{k}_{3}}{2}=\mathbf{k}_{1}+\frac{\mathbf{k}_{2}}{2}, \\
\mathbf{k}_{(13) 2} & =\sqrt{\frac{1}{3}}\left(\frac{\mathbf{k}_{1}+\mathbf{k}_{3}}{2}-\mathbf{k}_{2}\right)=-\sqrt{\frac{4}{3}} \mathbf{k}_{2} .
\end{aligned}
$$

The stationary states of three-body dynamics in finite volume are described by homogeneous Faddeev-type equations (see [50-53]),

$$
\begin{aligned}
\mathcal{T}^{(2 b)}\left(\mathbf{k}_{1}, \mathbf{k}_{2}\right)= & -\frac{1}{L^{3}} \sum_{\mathbf{p}_{1}} \frac{\tau^{(2 b)}\left(\mathbf{k}_{13} ; \mathbf{p}_{1}+\frac{\mathbf{k}_{2}}{2}\right)}{m E-\frac{\mathbf{p}_{1}^{2}+\mathbf{k}_{2}^{2}+\left(\mathbf{p}_{1}+\mathbf{k}_{2}\right)^{2}}{2}} \\
& \times\left[2 \mathcal{T}^{(2 b)}\left(\mathbf{k}_{2}, \mathbf{p}_{1}\right)+\mathcal{T}^{(3 b)}\left(\mathbf{p}_{1}, \mathbf{k}_{2}\right)\right]
\end{aligned}
$$

and

$\mathcal{T}^{(3 b)}\left(\mathbf{k}_{1}, \mathbf{k}_{2}\right)=-\frac{1}{L^{6}} \sum_{\mathbf{p}_{1}, \mathbf{p}_{2}} \frac{\tau^{(3 b)}(\mathbf{K} ; \mathbf{P})}{m E-\frac{\mathbf{p}_{1}^{2}+\mathbf{p}_{2}^{2}+\left(\mathbf{p}_{1}+\mathbf{p}_{2}\right)^{2}}{2}} 3 \mathcal{T}^{(2 b)}\left(\mathbf{p}_{1}, \mathbf{p}_{2}\right)$,

where

$$
\left(\mathbf{p}_{1}, \mathbf{p}_{2}\right) \in \frac{2 \pi \mathbf{n}}{L}, \quad \mathbf{n} \in \mathbb{Z}^{3} .
$$

The symbol $(\mathbf{K}, \mathbf{P})$ stand for 6-dimensional vectors; they are related to relative momenta $\left(\mathbf{k}_{13}, \mathbf{k}_{(13) 2}\right)$ by

$$
\begin{aligned}
& \mathbf{K}=\left\{\mathbf{k}_{13}, \mathbf{k}_{(13) 2}\right\}=\left\{\mathbf{k}_{1}+\frac{\mathbf{k}_{2}}{2},-\sqrt{\frac{4}{3}} \mathbf{k}_{2}\right\}, \\
& \mathbf{P}=\left\{\mathbf{p}_{13}, \mathbf{p}_{(13) 2}\right\}=\left\{\mathbf{p}_{1}+\frac{\mathbf{p}_{2}}{2},-\sqrt{\frac{4}{3}} \mathbf{p}_{2}\right\} .
\end{aligned}
$$

The length of $6 \mathrm{D}$ vectors is given by

$$
\begin{aligned}
& K=\sqrt{\mathbf{k}_{13}^{2}+\mathbf{k}_{(13) 2}^{2}}=\sqrt{\frac{1}{2} \sum_{i=1}^{3} \mathbf{k}_{i}^{2}}, \\
& P=\sqrt{\mathbf{p}_{13}^{2}+\mathbf{p}_{(13) 2}^{2}}=\sqrt{\frac{1}{2} \sum_{i=1}^{3} \mathbf{p}_{i}^{2}} .
\end{aligned}
$$

The symbols $\tau^{(2 b)}$ and $\tau^{(3 b)}$, which are associated with twobody interaction $V^{(2 b)}$ and three-body interaction $V^{(3 b)}$, respectively, are used to describe off-shell subprocess transition amplitudes between initial and final momenta states. For example, $\tau^{(2 b)}$ in the (13) isobar channel, with particle 2 carrying a momentum $\mathbf{k}_{2}$, satisfies two-body inhomogeneous Lippmann-Schwinger equations,

$$
\begin{aligned}
\tau^{(2 b)}\left(\mathbf{k}_{13} ; \mathbf{k}_{13}^{\prime}\right)= & -m \tilde{V}^{(2 b)}\left(\left|\mathbf{k}_{13}-\mathbf{k}_{13}^{\prime}\right|\right) \\
& +\frac{1}{L^{3}} \sum_{\mathbf{p}_{1}} \frac{m \tilde{V}^{(2 b)}\left(\left|\mathbf{k}_{13}-\mathbf{p}_{1}-\frac{\mathbf{k}_{2}}{2}\right|\right)}{m E-\frac{\mathbf{p}_{1}^{2}+\mathbf{k}_{2}^{2}+\left(\mathbf{p}_{1}+\mathbf{k}_{2}\right)^{2}}{2}} \\
& \times \tau^{(2 b)}\left(\mathbf{p}_{1}+\frac{\mathbf{k}_{2}}{2} ; \mathbf{k}_{13}^{\prime}\right),
\end{aligned}
$$

and similarly $\tau^{(3 b)}$ satisfies a three-body equation,

$$
\begin{aligned}
\tau^{(3 b)}\left(\mathbf{K} ; \mathbf{K}^{\prime}\right)= & -m \tilde{V}^{(3 b)}\left(\left|\mathbf{K}-\mathbf{K}^{\prime}\right|\right) \\
& +\frac{1}{L^{6}} \sum_{\mathbf{p}_{1}, \mathbf{p}_{2}} \frac{m \tilde{V}^{(3 b)}(|\mathbf{K}-\mathbf{P}|)}{m E-\frac{\mathbf{p}_{1}^{2}+\mathbf{p}_{2}^{2}+\left(\mathbf{p}_{1}+\mathbf{p}_{2}\right)^{2}}{2}} \tau^{(3 b)}\left(\mathbf{P} ; \mathbf{K}^{\prime}\right) .
\end{aligned}
$$

Here, $\tau^{(2 b)}$ and $\tau^{(3 b)}$ are the dynamical input of finite volume Faddeev equations in Eqs. (3) and (4), and must be solved first.

The quantization condition without the cubic irreducible representation projection is given by 


$$
\begin{aligned}
0= & \operatorname{det}\left[L^{6} \delta_{\mathbf{k}_{1}, \mathbf{p}_{1}} \delta_{\mathbf{k}_{2}, \mathbf{p}_{2}}+\frac{L^{3} \delta_{\mathbf{k}_{2}, \mathbf{p}_{1}} 2 \tau^{(2 b)}\left(\mathbf{k}_{13} ; \mathbf{p}_{2}+\frac{\mathbf{k}_{2}}{2}\right)}{m E-\frac{3 \mathbf{k}_{2}^{2}}{4}-\left(\mathbf{p}_{2}+\frac{\mathbf{k}_{2}}{2}\right)^{2}}\right. \\
& -\frac{3}{L^{3}} \sum_{\mathbf{p}} \frac{\tau^{(2 b)}\left(\mathbf{k}_{13} ; \mathbf{p}+\frac{\mathbf{k}_{2}}{2}\right) \tau^{(3 b)}\left(\left\{\mathbf{p}+\frac{\mathbf{k}_{2}}{2},-\sqrt{\frac{4}{3}} \mathbf{k}_{2}\right\} ; \mathbf{P}\right)}{\left[m E-\frac{3 \mathbf{k}_{2}^{2}}{4}-\left(\mathbf{p}+\frac{\mathbf{k}_{2}}{2}\right)^{2}\right]\left(m E-\mathbf{P}^{2}\right)},
\end{aligned}
$$

where $\tau^{(2 b)}$ and $\tau^{(3 b)}$, in principle, are given by the solutions of Eqs. (7) and (8), respectively. In Sec. II A, we show that with a separable interaction approximation, Eqs. (7) and (8) may be converted into algebraic equations. Hence, the solutions of $\tau^{(2 b)}$ and $\tau^{(3 b)}$ are Lüscher formulalike and can be formulated in terms of conventional two-body phase shifts in $3 \mathrm{D}$ and unconventional but mathematically convenient three-body phase shifts in $6 \mathrm{D}$. We remark that the momentum sum in the quantization condition must be regulated in the numerical evaluation of discrete energy levels, which are either associated with ultraviolet divergence or normalization of the determinant condition. In our current work, since the technical regularization is not our focus, the specific procedure of regularization has been left out, and we refer interested readers to Refs. [28,29].

\section{A. Separable interactions and algebraic solutions of $\tau^{(2 b)}$ and $\tau^{(3 b)}$}

Under the assumption of separable short-range potentials for both $V^{(2 b)}$ and $V^{(3 b)}$, the partial wave expansion of potentials have the forms

$$
\begin{aligned}
& \tilde{V}^{(2 b)}\left(\left|\mathbf{k}_{13}-\mathbf{k}_{13}^{\prime}\right|\right) \\
& =\sum_{L M} Y_{L M}\left(\hat{\mathbf{k}}_{13}\right) g_{L}^{(2 b)}\left(k_{13}\right) V_{L}^{(2 b)} g_{L}^{(2 b)}\left(k_{13}^{\prime}\right) Y_{L M}^{*}\left(\hat{\mathbf{k}}_{13}^{\prime}\right)
\end{aligned}
$$

and

$$
\begin{aligned}
& \tilde{V}^{(3 b)}(|\mathbf{K}-\mathbf{K}|) \\
& =\sum_{[J]} Y_{[J]}(\hat{\mathbf{K}}) g_{[J]}^{(3 b)}(K) V_{J}^{(3 b)} g_{[J]}^{(3 b)}\left(K^{\prime}\right) Y_{[J]}^{*}\left(\hat{\mathbf{K}}^{\prime}\right),
\end{aligned}
$$

where $Y_{L M}\left(\hat{\mathbf{k}}_{13}\right)$ is a 3D spherical harmonic function with quantum numbers $|L M\rangle$ representing orbital angular momentum configurations between particles 1 and 3 , while particle 2 acts as a spectator and is not involved in the interaction. Here, $Y_{[J]}(\hat{\mathbf{K}})$ stands for the $6 \mathrm{D}$ hyperspherical harmonic basis function (see Refs. [55,56] and also Appendix), and the quantum numbers $[J]$ represent a specific angular momentum configuration of three particles with a total angular momentum $J$. Note that $Y_{[J]}(\hat{\mathbf{K}})$ may be constructed through two 3D spherical harmonic functions. For example, consider a configuration with angular momentum state $\left|L_{13} M_{13}\right\rangle$ between particles 1 and 3, coupled with particle 2 in relative angular momentum state
$\left|L_{(13) 2} M_{(13) 2}\right\rangle$ into total angular momentum state $|[J]\rangle=\left|J M L_{13} L_{(13) 2}\right\rangle$; thus, $Y_{[J]}(\hat{\mathbf{K}})$ is given by

$$
\begin{aligned}
Y_{[J]}(\hat{\mathbf{K}})= & \sum_{M_{13}, M_{(13) 2}}\left\langle L_{13} M_{13}, L_{(13) 2} M_{(13) 2} \mid J M\right\rangle \\
& \times Y_{L_{13} M_{13}}\left(\hat{\mathbf{k}}_{13}\right) Y_{L_{(13) 2} M_{(13) 2}}\left(\hat{\mathbf{k}}_{(13) 2}\right) \mathcal{P}_{J L_{13} L_{(13) 2}}(\phi),
\end{aligned}
$$

where

$$
\phi=\tan ^{-1} \frac{k_{13}}{k_{(13) 2}} .
$$

The function $\mathcal{P}_{J L_{13} L_{(13) 2}}(\phi)$ is related to the Jacobi polynomial by (see also $[55,56]$ )

$$
\begin{aligned}
& \mathcal{P}_{J L_{13} L_{(13) 2}}(\phi)=N_{J L_{13} L_{(13) 2}}(\sin \phi)^{L_{13}}(\cos \phi)^{L_{(13) 2}} \\
& \times P_{\frac{J-L_{13}-L_{(13) 2}}{2}}^{\left(L_{13}+\frac{1}{2}, L_{(13)}+\frac{1}{2}\right)}(\cos 2 \phi),
\end{aligned}
$$

and the normalization factor $N_{J L_{13} L_{(13) 2}}$ is determined by the orthonormal relation

$$
\int_{0}^{\frac{\pi}{2}} d \phi \sin ^{2} \phi \cos ^{2} \phi \mathcal{P}_{J L_{13} L_{(13) 2}}(\phi) \mathcal{P}_{J^{\prime} L_{13} L_{(13) 2}}(\phi)=\delta_{J, J^{\prime}}
$$

The form factors $g_{L}^{(2 b)}$ and $g_{[J]}^{(3 b)}$ and potential strengths $V_{L}^{(2 b)}$ and $V_{J}^{(3 b)}$ may be considered as model parameters. Usually, the form factors, such as $g_{L}^{(2 b)}$, must show the correct threshold behavior,

$$
g_{L}^{(2 b)}(k \rightarrow 0) \sim k^{L} .
$$

The potential strengths $V_{L}^{(2 b)}$ and $V_{J}^{(3 b)}$ may be used to model two-body and three-body resonances; for example, the two-particle resonance of mass $m_{R}^{(2 b)}$ in the (13) isobar pair channel with particle 2 carrying momentum $\mathbf{k}_{2}$ may be given by

$$
V_{L}^{(2 b)} \propto \frac{1}{\left(E-\frac{3}{4} \frac{\mathbf{k}_{2}^{2}}{m}\right)-m_{R}^{(2 b)}} .
$$

A three-particle resonance of mass $m_{R}^{(3 b)}$ thus may be modeled similarly by

$$
V_{L}^{(3 b)} \propto \frac{1}{E-m_{R}^{(3 b)}} .
$$

Separable interactions suggest that $\tau^{(2 b)}$ in Eq. (7) and $\tau^{(3 b)}$ in Eq. (8) may be given by Lüscher formulalike algebra equations (see detailed discussion in Appendix), 


$$
\begin{aligned}
& \frac{\sqrt{m E-\frac{3}{4} \mathbf{k}_{2}^{2}}}{16 \pi^{2}} \tau^{(2 b)}\left(\mathbf{k}_{13} ; \mathbf{k}_{13}^{\prime}\right) \\
& \left.=\sum_{L M, L^{\prime} M^{\prime}} \frac{Y_{L M}\left(\hat{k}_{13}\right) g_{L}^{(2 b)}\left(k_{13}\right) g_{L^{\prime}}^{(2 b)}\left(k_{13}^{\prime}\right) Y_{L^{\prime} M^{\prime}}^{*}\left(\hat{k}_{13}^{\prime}\right)}{m E-\frac{3}{4} \mathbf{k}_{2}^{2}}\right) g_{L^{\prime}}^{(2 b)}\left(\sqrt{m E-\frac{3}{4} \mathbf{k}_{2}^{2}}\right) \\
& \quad \times i^{L-L^{\prime}}\left[\mathbb{I} \cdot \cot \delta^{(2 b)}\left(\sqrt{m E-\frac{3}{4} \mathbf{k}_{2}^{2}}\right)\right. \\
& \left.-\mathcal{M}^{\left(2 b, \mathbf{k}_{2}\right)}\left(\sqrt{m E-\frac{3}{4} \mathbf{k}_{2}^{2}}\right)\right]_{L M, L^{\prime} M^{\prime}}^{-1},
\end{aligned}
$$

and

$$
\begin{aligned}
& \frac{(m E)^{2}}{128 \pi^{5}} \tau^{(3 b)}\left(\mathbf{K} ; \mathbf{K}^{\prime}\right) \\
& =\sum_{[J],\left[J^{\prime}\right]} \frac{Y_{[J]}(\hat{K}) g_{[J]}^{(3 b)}(K) g_{\left[J^{\prime}\right]}^{(3 b)}\left(K^{\prime}\right) Y_{\left[J^{\prime}\right]}^{*}\left(\hat{K}^{\prime}\right)}{g_{[J]}^{(3 b)}(\sqrt{m E}) g_{\left[J^{\prime}\right]}^{(3 b)}(\sqrt{m E})} \\
& \quad \times i^{J-J^{\prime}}\left[\mathbb{I} \cdot \cot \delta^{(3 b)}(\sqrt{m E})-\mathcal{M}^{(3 b)}(\sqrt{m E})\right]_{[J],\left[J^{\prime}\right]}^{-1} .
\end{aligned}
$$

Generalized Lüscher zeta functions, $\mathcal{M}^{\left(2 b, \mathbf{k}_{2}\right)}$ in $3 \mathrm{D}$ and $\mathcal{M}^{(3 b)}$ in $6 \mathrm{D}$, are given, respectively, by

$$
\begin{aligned}
& \frac{k}{16 \pi^{2}} \mathcal{M}_{L M, L^{\prime} M^{\prime}}^{\left(2 b, \mathbf{k}_{2}\right)}(k)=\delta_{L M, L^{\prime} M^{\prime}} \frac{i k}{16 \pi^{2}} \\
& \quad+\delta_{L M, L^{\prime} M^{\prime}} \int \frac{p^{2} d p}{(2 \pi)^{3}}\left(\frac{g_{L}^{(2 b)}(p)}{g_{L}^{(2 b)}(k)}\right)^{2} \frac{1}{k^{2}-p^{2}} \\
& -\frac{1}{L^{3}} \sum_{\mathbf{p}=\frac{2 \pi \mathbf{n}}{L}+\frac{\mathbf{k}_{2}}{2}, \mathbf{n} \in \mathbb{Z}^{3}} \frac{g_{L}^{(2 b)}(p) g_{L^{\prime}}^{(2 b)}(p)}{g_{L}^{(2 b)}(k) g_{L^{\prime}}^{(2 b)}(k)} \frac{Y_{L M}^{*}(\hat{\mathbf{p}}) Y_{L^{\prime} M^{\prime}}(\hat{\mathbf{p}})}{k^{2}-\mathbf{p}^{2}}
\end{aligned}
$$

and

$$
\begin{aligned}
& \frac{(m E)^{2}}{128 \pi^{5}} \mathcal{M}_{[J],\left[J^{\prime}\right]}^{(3 b)}(\sqrt{m E})=\delta_{[J],\left[J^{\prime}\right]} \frac{i(m E)^{2}}{128 \pi^{5}} \\
& \quad+\delta_{[J],\left[J^{\prime}\right]} \int \frac{P^{5} d P}{(2 \pi)^{6}}\left(\frac{g_{J}^{(3 b)}(P)}{g_{J}^{(3 b)}(\sqrt{m E})}\right)^{2} \frac{1}{m E-P^{2}} \\
& \quad-\frac{1}{L^{6}} \sum_{\mathbf{p}_{1}, \mathbf{p}_{2}} \frac{g_{J}^{(3 b)}(P) g_{J^{\prime}}^{(3 b)}(P)}{g_{J}^{(3 b)}(\sqrt{m E}) g_{J^{\prime}}^{(3 b)}(\sqrt{m E})} \frac{Y_{[J]}^{*}(\hat{\mathbf{P}}) Y_{\left[J^{\prime}\right]}(\hat{\mathbf{P}})}{m E-P^{2}}
\end{aligned}
$$

The two-body phase shift $\delta_{L}^{(2 b)}$ is defined in a conventional way, which may be modeled and constrained by experimental data. The unconventional three-body phase shift $\delta_{J}^{(3 b)}$ may be interpreted as scattering of one particle off a short-range potential in $6 \mathrm{D}$. It may only serve as a mathematically convenient tool for modeling of the three-body resonance of total spin $J$.

\section{B. Quantization condition with separable interactions approximation}

Algebraic solutions of $\tau^{(2 b)}$ in Eq. (18) and $\tau^{(3 b)}$ in Eq. (19) suggest that the partial expansion of $\mathcal{T}^{(2 b)}\left(\mathbf{k}_{1}, \mathbf{k}_{2}\right)$ may have the form

$$
\mathcal{T}^{(2 b)}\left(\mathbf{k}_{1}, \mathbf{k}_{2}\right)=\sum_{L M} Y_{L M}\left(\hat{\mathbf{k}}_{13}\right) g_{L}^{(2 b)}\left(k_{13}\right) \mathcal{T}_{L M}^{(2 b)}\left(\mathbf{k}_{2}\right)
$$

The separable form $\mathcal{T}^{(2 b)}\left(\mathbf{k}_{1}, \mathbf{k}_{2}\right)$ thus allows one to further reduce Faddeev equations, Eqs. (3) and (4), to

$$
\begin{aligned}
\mathcal{T}_{L M}^{(2 b)}\left(\mathbf{k}_{2}\right)= & -\frac{1}{L^{3}} \sum_{\mathbf{p}_{2}} \sum_{L^{\prime} M^{\prime}} \frac{2 \tau_{L M}^{(2 b)}\left(\mathbf{p}_{2}+\frac{\mathbf{k}_{2}}{2}\right) g_{L^{\prime}}^{(2 b)}\left(\left|\mathbf{k}_{2}+\frac{\mathbf{p}_{2}}{2}\right|\right) Y_{L^{\prime} M^{\prime}}\left(\mathbf{k}_{2}+\frac{\mathbf{p}_{2}}{2}\right)}{m E-\frac{3}{4} \mathbf{k}_{2}^{2}-\left(\mathbf{p}_{2}+\frac{\mathbf{k}_{2}}{2}\right)^{2}} \mathcal{T}_{L^{\prime} M^{\prime}}^{(2 b)}\left(\mathbf{p}_{2}\right) \\
& +\frac{1}{L^{3}} \sum_{\mathbf{p}_{2}} \sum_{L^{\prime} M^{\prime}}\left[\frac{3}{L^{6}} \sum_{\mathbf{k}_{1}, \mathbf{p}_{1}} \frac{\tau_{L M}^{(2 b)}\left(\mathbf{k}_{13}\right)}{m E-\mathbf{K}^{2}} \frac{\tau^{(3 b)}(\mathbf{K} ; \mathbf{P}) g_{L^{\prime}}^{(2 b)}\left(p_{13}\right) Y_{L^{\prime} M^{\prime}}\left(\hat{\mathbf{p}}_{13}\right)}{m E-\mathbf{P}^{2}}\right] \mathcal{T}_{L^{\prime} M^{\prime}}^{(2 b)}\left(\mathbf{p}_{2}\right),
\end{aligned}
$$

where $\tau_{L M}^{(2 b)}\left(\mathbf{k}_{13}^{\prime}\right)$ is defined by the relation

$$
\tau^{(2 b)}\left(\mathbf{k}_{13} ; \mathbf{k}_{13}^{\prime}\right)=\sum_{L M} Y_{L M}\left(\hat{\mathbf{k}}_{13}\right) g_{L}^{(2 b)}\left(k_{13}\right) \tau_{L M}^{(2 b)}\left(\mathbf{k}_{13}^{\prime}\right) .
$$

Therefore, a partially expanded quantization condition is given by

$$
\begin{gathered}
\operatorname{det}\left[\delta_{L M, L^{\prime} M^{\prime}} L^{3} \delta_{\mathbf{k}_{2}, \mathbf{p}_{2}}+\frac{2 \tau_{L M}^{(2 b)}\left(\mathbf{p}_{2}+\frac{\mathbf{k}_{2}}{2}\right) g_{L^{\prime}}^{(2 b)}\left(\left|\mathbf{k}_{2}+\frac{\mathbf{p}_{2}}{2}\right|\right) Y_{L^{\prime} M^{\prime}}\left(\mathbf{k}_{2}+\frac{\mathbf{p}_{2}}{2}\right)}{m E-\frac{3}{4} \mathbf{k}_{2}^{2}-\left(\mathbf{p}_{2}+\frac{\mathbf{k}_{2}}{2}\right)^{2}}\right. \\
\left.-\frac{1}{L^{6}} \sum_{\mathbf{k}_{1}, \mathbf{p}_{1}} \frac{3 \tau_{L M}^{(2 b)}\left(\mathbf{k}_{13}\right) \tau^{(3 b)}(\mathbf{K} ; \mathbf{P}) g_{L^{\prime}}^{(2 b)}\left(p_{13}\right) Y_{L^{\prime} M^{\prime}}\left(\hat{\mathbf{p}}_{13}\right)}{\left(m E-\mathbf{K}^{2}\right)\left(m E-\mathbf{P}^{2}\right)}\right]=0
\end{gathered}
$$


As a specific example, let us consider a simple case with only $S$-wave contributions in both two-body and threebody channels, that is to say, $J=L_{13}=L_{(13) 2}=0$. Thus, $\tau^{(2 b)}$ and $\tau^{(3 b)}$ are given, respectively, by phase shifts $\delta_{L_{13}=0}^{(2 b)}$ and $\delta_{J=0}^{(3 b)}$ only,

$$
\begin{gathered}
\tau^{(2 b)}\left(\mathbf{k}_{13} ; \mathbf{k}_{13}^{\prime}\right)=\tau^{\left(2 b, \mathbf{k}_{2}\right)}\left(\sqrt{m E-\frac{3}{4} \mathbf{k}_{2}^{2}}\right)=\frac{4 \pi}{\sqrt{m E-\frac{3}{4} \mathbf{k}_{2}^{2}}} \\
\times \frac{1}{\cot \delta_{0}^{(2 b)}\left(\sqrt{m E-\frac{3}{4} \mathbf{k}_{2}^{2}}\right)-\mathcal{M}_{00,00}^{\left(2 b, \mathbf{k}_{2}\right)}\left(\sqrt{m E-\frac{3}{4} \mathbf{k}_{2}^{2}}\right)}
\end{gathered}
$$

and

$$
\begin{aligned}
\tau^{(3 b)}\left(\mathbf{K} ; \mathbf{K}^{\prime}\right) & =\tau^{(3 b)}(\sqrt{m E}) \\
& =\frac{128 \pi^{2}}{(m E)^{2}} \frac{1}{\cot \delta_{0}^{(3 b)}(\sqrt{m E})-\mathcal{M}_{[0],[0]}^{(3 b)}(\sqrt{m E})} .
\end{aligned}
$$

The quantization condition in this case is given by a simple form,

$$
\begin{aligned}
& \operatorname{det}\left[L^{3} \delta_{\mathbf{k}_{2}, \mathbf{p}_{2}}+\frac{2 \tau^{\left(2 b, \mathbf{k}_{2}\right)}\left(\sqrt{m E-\frac{3}{4} \mathbf{k}_{2}^{2}}\right)}{m E-\frac{3}{4} \mathbf{k}_{2}^{2}-\left(\mathbf{p}_{2}+\frac{\mathbf{k}_{2}}{2}\right)^{2}}\right. \\
& \left.+\frac{1}{L^{6}} \sum_{\mathbf{k}_{1}, \mathbf{p}_{1}} \frac{3 \tau^{\left(2 b, \mathbf{k}_{2}\right)}\left(\sqrt{m E-\frac{3}{4} \mathbf{k}_{2}^{2}}\right) \tau^{(3 b)}(\sqrt{m E})}{\left(m E-\mathbf{K}^{2}\right)\left(m E-\mathbf{P}^{2}\right)}\right]=0 .
\end{aligned}
$$

The two-body and three-body resonances hence can be inserted through modeling of $\delta_{L_{13}=0}^{(2 b)}$ and $\delta_{J=0}^{(3 b)}$.

\section{SUMMARY}

In summary, with the separable interaction approximation, we show that the subprocess transition amplitudes are Lüscher formulalike, and the quantization condition may be formulated in terms of both two-body and three-body phase shifts that may be useful for describing resonances in fewbody interactions. Two-body phase shifts may be modeled and constrained by experimental data, and three-body phase shifts may serve as a convenient tool for inserting three-body resonances with a specific spin into the quantization condition.

\section{ACKNOWLEDGMENTS}

We acknowledge support from the Department of Physics and Engineering, California State University,
Bakersfield. This research was supported in part by the National Science Foundation under Grant No. NSF PHY1748958.

\section{APPENDIX: LÜSCHER FORMULA IN $D$-DIMENSIONAL SPACE}

\section{Scattering in $D$-dimensional space}

Let us start with an $N$-body Schrödinger equation in the center-of-mass frame,

$$
\left[m E+\sum_{j=1}^{N-1} \nabla_{\boldsymbol{\xi}_{j}}^{2}\right] \psi(\boldsymbol{\xi} ; \mathbf{K})=m V(\xi) \psi(\boldsymbol{\xi} ; \mathbf{K}) .
$$

The relative coordinates of the $N$-particle are given by

$$
\begin{aligned}
\boldsymbol{\xi}_{j} & =\sqrt{\frac{2 j}{j+1}}\left(\frac{1}{j} \sum_{i=1}^{j} \mathbf{x}_{i}-\mathbf{x}_{j+1}\right), \\
\mathbf{q}_{j} & =\sqrt{\frac{j}{2(j+1)}}\left(\frac{1}{j} \sum_{i=1}^{j} \mathbf{k}_{i}-\mathbf{k}_{j+1}\right), \quad j=1, \ldots, \quad N-1,
\end{aligned}
$$

where $\mathbf{x}_{i}$ and $\mathbf{k}_{i}$ stand for the coordinate and momentum of the $i$ th particle, respectively. Here, the $D=3(N-1)$ dimensional vectors $(\boldsymbol{\xi}, \mathbf{K})$ are defined by the relative coordinates and momenta of the particles,

$$
\begin{gathered}
\boldsymbol{\xi}=\left\{\boldsymbol{\xi}_{1}, \boldsymbol{\xi}_{2}, \ldots, \boldsymbol{\xi}_{N-1}\right\}, \quad \boldsymbol{\xi}=|\boldsymbol{\xi}|=\sqrt{\sum_{j=1}^{N-1} \boldsymbol{\xi}_{j}^{2}}, \\
\mathbf{K}=\left\{\mathbf{q}_{1}, \mathbf{q}_{2}, \ldots, \mathbf{q}_{N-1}\right\} \quad, K=|\mathbf{K}|=\sqrt{\sum_{j=1}^{N-1} \mathbf{q}_{j}^{2} .}
\end{gathered}
$$

Note that the $D$-dimensional Laplace operator has a separable form between the radial and orbital terms [55,56],

$$
\nabla_{D}^{2}=\sum_{j=1}^{N-1} \nabla_{\xi_{j}}^{2}=\frac{1}{\xi^{D-1}} \frac{\partial}{\partial \xi} \xi^{D-1} \frac{\partial}{\partial \xi}+\frac{\hat{L}^{2}\left(\Omega_{D}\right)}{\xi^{2}},
$$

where $\hat{L}^{2}\left(\Omega_{D}\right)$ is the grand orbital operator. The eigenstates of the orbital equation

$$
\hat{L}^{2}\left(\Omega_{D}\right) Y_{[L]}\left(\Omega_{D}\right)=L(L+D-2) Y_{[L]}\left(\Omega_{D}\right)
$$

are given by the hyperspherical harmonic $Y_{[L]}\left(\Omega_{D}\right)[55,56]$, where $[L]$ is a set of $D-1$ quantum numbers, including total orbital angular momentum $L$. The hyperspherical harmonic $Y_{[L]}\left(\Omega_{D}\right)$ basis defines a complete set of orthonormal angular functions in $D$-dimensional space, 


$$
\int d \Omega_{D} Y_{[L]}^{*}\left(\Omega_{D}\right) Y_{\left[L^{\prime}\right]}\left(\Omega_{D}\right)=\delta_{[L],\left[L^{\prime}\right]} .
$$

The scattering in $D$-dimensional space can also be described by the Lippmann-Schwinger equations

$$
\begin{aligned}
\psi(\boldsymbol{\xi} ; \mathbf{K}) & =e^{i \mathbf{K} \cdot \boldsymbol{\xi}}+\int d \boldsymbol{\xi}^{\prime} G_{D}\left(\boldsymbol{\xi}-\boldsymbol{\xi}^{\prime} ; E\right) m V\left(\xi^{\prime}\right) \psi\left(\boldsymbol{\xi}^{\prime} ; \mathbf{K}\right), \\
G_{D}\left(\boldsymbol{\xi}-\boldsymbol{\xi}^{\prime} ; E\right) & =\int \frac{d \mathbf{Q}}{(2 \pi)^{D}} \frac{e^{i \mathbf{Q} \cdot\left(\boldsymbol{\xi}-\boldsymbol{\xi}^{\prime}\right)}}{m E-\mathbf{Q}^{2}},
\end{aligned}
$$

where the Green's function satisfies the equation

$$
\left[m E+\nabla_{D}^{2}\right] G_{D}\left(\boldsymbol{\xi}-\boldsymbol{\xi}^{\prime} ; E\right)=\delta\left(\boldsymbol{\xi}-\boldsymbol{\xi}^{\prime}\right) .
$$

The analytic expression of the Green's function and its partial wave expansion in terms of the hyperspherical harmonic basis are given, respectively, by

$$
G_{D}(\xi ; E)=-\frac{i}{4} \frac{(m E)^{\frac{D}{2}-1}}{(2 \pi)^{\frac{D}{2}-1}} \frac{H_{\frac{D}{2}-1}^{(1)}(\sqrt{m E} \xi)}{(\sqrt{m E} \xi)^{\frac{D}{2}-1}}
$$

and

$$
\begin{aligned}
& G_{D}\left(\boldsymbol{\xi}-\boldsymbol{\xi}^{\prime} ; E\right)^{\xi \stackrel{\xi}{=} \xi^{\prime}}-i(m E)^{\frac{D-2}{2}} \\
& \quad \times \sum_{[L]} Y_{[L]}\left(\Omega_{\xi}\right) \mathcal{H}_{L}^{(1)}(\sqrt{m E} \xi) \mathcal{J}_{L}\left(\sqrt{m E} \xi^{\prime}\right) Y_{[L]}^{*}\left(\Omega_{\xi^{\prime}}\right),
\end{aligned}
$$

where

$$
\mathcal{J}_{L}(z)=\sqrt{\frac{\pi}{2}} \frac{J_{L+\frac{D-2}{2}}(z)}{z^{\frac{D}{2}-1}}, \quad \mathcal{N}_{L}(z)=\sqrt{\frac{\pi}{2}} \frac{N_{L+\frac{D-2}{2}}(z)}{z^{\frac{D}{2}-1}},
$$

and

$$
\mathcal{H}_{L}^{(1)}(z)=\mathcal{J}_{L}(z)+i \mathcal{N}_{L}(z)
$$

Assuming that the potential $V(\xi)$ is spherical and shortranged, and also using partial wave expansion of the plane wave in $D$-dimensional space,

$$
e^{i \mathbf{K} \cdot \xi}=\sqrt{\frac{2}{\pi}}(2 \pi)^{\frac{D}{2}} \sum_{[L]} i^{L} Y_{[L]}\left(\Omega_{\xi}\right) Y_{[L]}^{*}\left(\Omega_{\mathbf{K}}\right) \mathcal{J}_{L}(\sqrt{m E} \xi),
$$

the asymptotic form of the wave function is obtained,

$$
\begin{aligned}
& \psi(\xi ; \mathbf{K}) \stackrel{\text { Large } \xi}{\rightarrow} \sqrt{\frac{2}{\pi}}(2 \pi)^{\frac{D}{2}} \sum_{[L]} i^{L} Y_{[L]}\left(\Omega_{\xi}\right) Y_{[L]}^{*}\left(\Omega_{\mathbf{K}}\right) \\
& \quad \times\left[\mathcal{J}_{L}(\sqrt{m E} \xi)+i f_{L}^{(D)}(\sqrt{m E}) \mathcal{H}_{L}^{(1)}(\sqrt{m E} \xi)\right],
\end{aligned}
$$

where $f_{L}^{(D)}$ is defined by

$$
\begin{aligned}
& \sqrt{\frac{2}{\pi}} \frac{(2 \pi)^{\frac{D}{2}}}{(m E)^{\frac{D-2}{2}}} i^{L} f_{L}^{(D)}(\sqrt{m E}) Y_{[L]}^{*}\left(\Omega_{\mathbf{K}}\right) \\
& =-\int d \boldsymbol{\xi}^{\prime} Y_{[L]}^{*}\left(\Omega_{\xi^{\prime}}\right) \mathcal{J}_{L}\left(\sqrt{m E} \xi^{\prime}\right) m V\left(\xi^{\prime}\right) \psi\left(\boldsymbol{\xi}^{\prime} ; \mathbf{K}\right) .
\end{aligned}
$$

Thus, $f_{L}^{(D)}$ may be interpreted as the partial wave scattering amplitude in $D$-dimensional space, and it can be parametrized in terms of the $D$-dimensional phase shift $\delta_{L}^{(D)}\left(k_{E}\right)$ $[55,56]$ by

$$
f_{L}^{(D)}(\sqrt{m E})=\frac{1}{\cot \delta_{L}^{(D)}(\sqrt{m E})-i} .
$$

\section{Lippmann-Schwinger equation in momentum space and separable potential approximation}

The off-shell transition amplitude between initial and final momentum states $|\mathbf{K}\rangle$ and $\left|\mathbf{K}^{\prime}\right\rangle$ may be introduced by

$$
t^{(D)}\left(\mathbf{K}, \mathbf{K}^{\prime}\right)=-\int d \boldsymbol{\xi} e^{-i \mathbf{K}^{\prime} \cdot \boldsymbol{\xi}} m V(\xi) \psi(\boldsymbol{\xi} ; \mathbf{K}) .
$$

Thus, Eq. (A7) can be converted into a momentum-space Lippmann-Schwinger equation,

$$
\begin{aligned}
t^{(D)}\left(\mathbf{K}, \mathbf{K}^{\prime}\right)= & -m \tilde{V}\left(\left|\mathbf{K}-\mathbf{K}^{\prime}\right|\right) \\
& +\int \frac{d \mathbf{Q}}{(2 \pi)^{D}} \frac{m \tilde{V}(|\mathbf{K}-\mathbf{Q}|)}{m E-\mathbf{Q}^{2}} t^{(D)}\left(\mathbf{Q}, \mathbf{K}^{\prime}\right) .
\end{aligned}
$$

The partial wave expansion of the above equation yields

$$
\begin{aligned}
t_{L}^{(D)}\left(K, K^{\prime}\right)= & -m \tilde{V}_{L}\left(K, K^{\prime}\right) \\
& +\int \frac{Q^{D-1} d Q}{(2 \pi)^{D}} \frac{m \tilde{V}_{L}(K, Q)}{m E-\mathbf{Q}^{2}} t_{L}^{(D)}\left(Q, K^{\prime}\right),
\end{aligned}
$$

where the expansion relations of the potential and amplitude are given by

$$
\tilde{V}\left(\left|\mathbf{K}-\mathbf{K}^{\prime}\right|\right)=\sum_{[L]} Y_{[L]}(\hat{\mathbf{K}}) \tilde{V}_{L}\left(K, K^{\prime}\right) Y_{[L]}^{*}\left(\hat{\mathbf{K}}^{\prime}\right)
$$

and

$$
t^{(D)}\left(\mathbf{K}, \mathbf{K}^{\prime}\right)=\sum_{[L]} Y_{[L]}(\hat{\mathbf{K}}) t_{L}\left(K, K^{\prime}\right) Y_{[L]}^{*}\left(\hat{\mathbf{K}}^{\prime}\right) .
$$

Under the assumption of a separable potential,

$$
\tilde{V}_{L}\left(K, K^{\prime}\right)=g_{L}^{(D)}(K) V_{L} g_{L}^{(D)}\left(K^{\prime}\right),
$$


where $g_{L}^{(D)}$ and $V_{L}$ stand for the form factor and interaction strength of the potential; thus, a closed algebra form of the off-shell partial wave amplitude, $t_{L}^{(D)}\left(K, K^{\prime}\right)$, may be obtained (see [57]),

$$
t_{L}^{(D}\left(K, K^{\prime}\right)=-\frac{g_{L}^{(D)}(K) g_{L}^{(D)}\left(K^{\prime}\right)}{\frac{1}{m V_{L}}-\int \frac{Q^{D-1} d Q}{(2 \pi)^{D}} \frac{\left(g_{L}^{(D)}(Q)\right)^{2}}{m E-\mathbf{Q}^{2}}} .
$$

Compared with the on-shell scattering amplitude $f_{L}^{(D)}(\sqrt{m E})$ in Eq. (A15), we find

$$
\begin{aligned}
t_{L}^{(D}\left(K, K^{\prime}\right)= & \frac{g_{L}^{(D)}(K) g_{L}^{(D)}\left(K^{\prime}\right)}{\left(g_{L}^{(D)}(\sqrt{m E})\right)^{2}} \\
& \times \frac{2}{\pi} \frac{(2 \pi)^{D}}{(m E)^{\frac{D-2}{2}}} \frac{1}{\cot \delta_{L}^{(D)}(\sqrt{m E})-i},
\end{aligned}
$$

and also the useful relation

$$
\begin{aligned}
\frac{1}{m V_{L}}= & \int \frac{Q^{D-1} d Q}{(2 \pi)^{D}} \frac{\left(g_{L}^{(D)}(Q)\right)^{2}}{m E-\mathbf{Q}^{2}} \\
& +\left(g_{L}^{(D)}(\sqrt{m E})\right)^{2} \frac{\pi}{2} \frac{(m E)^{\frac{D-2}{2}}}{(2 \pi)^{D}}\left[i-\cot \delta_{L}^{(D)}(\sqrt{m E})\right]
\end{aligned}
$$

Therefore, the off-shell partial wave amplitude $t_{L}^{(D)}\left(K, K^{\prime}\right)$ may be modeled in terms of the on-shell physical quantity: phase shifts $\delta_{L}^{(D)}(\sqrt{m E})$.

We remark that the separable potential approximation is in fact based on the assumption of a hyperspherical shortrange interaction. The hyperspherical partial wave expansion of the momentum-space potential is given by

$$
\begin{aligned}
\tilde{V}\left(\left|\mathbf{K}-\mathbf{K}^{\prime}\right|\right)= & \int d \boldsymbol{\xi} e^{-i\left(\mathbf{K}-\mathbf{K}^{\prime}\right) \cdot \xi} V(\xi) \\
\propto & \sum_{[L]} Y_{[L]}(\hat{\mathbf{K}}) \int \xi^{D-1} d \xi \mathcal{J}_{L}(K \xi) V(\xi) \\
& \times \mathcal{J}_{L}\left(K^{\prime} \xi\right) Y_{[L]}^{*}\left(\hat{\mathbf{K}}^{\prime}\right) .
\end{aligned}
$$

For a short-range potential, asymptotically one obtains

$$
\int \xi^{D-1} d \xi \mathcal{J}_{L}(K \xi) V(\xi) \mathcal{J}_{L}\left(K^{\prime} \xi\right) \sim K^{L} V_{L} K^{\prime L},
$$

which thus yields the expression in Eq. (A22). The separable potential approximation may be useful for modeling sharp few-body resonances that are predominantly generated by quark and gluon dynamics. Hence, the hadron-hadron interactions may be well approximated by a short-range energy-dependent interaction, and the
Breit-Wigner formula is a good example of such an approximation.

\section{Lüscher formula in $D$-dimensional space and separable potential approximation}

The scattering solution in finite volume may be described by an inhomogeneous Lippmann-Schwinger equation,

$$
\begin{aligned}
\tau^{(D)}\left(\mathbf{K}, \mathbf{K}^{\prime}\right)= & -m \tilde{V}\left(\left|\mathbf{K}-\mathbf{K}^{\prime}\right|\right) \\
& +\frac{1}{L^{D}} \sum_{\mathbf{p}_{1}, \ldots, \mathbf{p}_{N-1}} \frac{m \tilde{V}(|\mathbf{K}-\mathbf{Q}|)}{m E-\mathbf{Q}^{2}} \tau^{(D)}\left(\mathbf{Q}, \mathbf{K}^{\prime}\right),
\end{aligned}
$$

where $\mathbf{p}_{i} \in \frac{2 \pi \mathbf{n}}{L}, \mathbf{n} \in \mathbb{Z}^{3}$, and $\mathbf{Q}^{2}=\frac{1}{2} \sum_{i=1}^{N} \mathbf{p}_{i}^{2}$. Considering the partial wave expansion again,

$$
\tau^{(D)}\left(\mathbf{K}, \mathbf{K}^{\prime}\right)=\sum_{[L],\left[L^{\prime}\right]} Y_{[L]}(\hat{\mathbf{K}}) \tau_{[L],\left[L^{\prime}\right]}^{(D)}\left(K, K^{\prime}\right) Y_{\left[L^{\prime}\right]}^{*}\left(\hat{\mathbf{K}}^{\prime}\right),
$$

one finds

$$
\begin{aligned}
\tau_{[L],\left[L^{\prime}\right]}^{(D)}\left(K, K^{\prime}\right) \\
=-\delta_{[L],\left[L^{\prime}\right]} m \tilde{V}_{L}\left(K, K^{\prime}\right) \\
\quad+\sum_{[l]} \frac{1}{L^{D}} \sum_{\mathbf{p}_{1}, \ldots, \mathbf{p}_{N-1}} \frac{m \tilde{V}_{L}(K, Q) Y_{[L]}^{*}(\hat{\mathbf{Q}}) Y_{[l]}(\hat{\mathbf{Q}})}{m E-\mathbf{Q}^{2}} \\
\quad \times \tau_{[l],\left[L^{\prime}\right]}^{(D)}\left(Q, K^{\prime}\right) .
\end{aligned}
$$

Again, the separable potential given in Eq. (A22) suggests that $\tau_{[L],\left[L^{\prime}\right]}^{(D)}$ may have the separable form

$$
\tau_{[L],\left[L^{\prime}\right]}^{(D)}\left(K, K^{\prime}\right)=g_{L}(K) C_{[L],\left[L^{\prime}\right]}(E) g_{L^{\prime}}\left(K^{\prime}\right),
$$

where $C_{[L],\left[L^{\prime}\right]}(E)$ satisfies a matrix equation,

$$
\begin{aligned}
C_{[L],\left[L^{\prime}\right]}(E)= & -\delta_{[L],\left[L^{\prime}\right]} m V_{L}+\sum_{[l]} \frac{1}{L^{D}} \sum_{\mathbf{p}_{1}, \ldots, \mathbf{p}_{N-1}} \\
& \times g_{L}^{(D)}(Q) g_{l}^{(D)}(Q) \frac{Y_{[L]}^{*}(\hat{\mathbf{Q}}) Y_{[l]}(\hat{\mathbf{Q}})}{m E-\mathbf{Q}^{2}} C_{[l],\left[L^{\prime}\right]}(E) .
\end{aligned}
$$

Hence, a closed algebra form of the off-shell solution of finite volume amplitude, $\tau_{[L],\left[L^{\prime}\right]}^{(D)}$, is obtained as

$$
\tau_{[L],\left[L^{\prime}\right]}^{(D)}\left(K, K^{\prime}\right)=\frac{g_{L}(K) g_{L^{\prime}}\left(K^{\prime}\right)}{g_{L}(\sqrt{m E}) g_{L^{\prime}}(\sqrt{m E})}[\mathcal{D}(\sqrt{m E})]_{[L],\left[L^{\prime}\right]}^{-1},
$$


where

$$
\begin{aligned}
\mathcal{D}_{[L],\left[L^{\prime}\right]}(\sqrt{m E}) \\
=-\frac{\delta_{[L],\left[L^{\prime}\right]}}{g_{L}(\sqrt{m E}) m V_{L} g_{L^{\prime}}(\sqrt{m E})} \\
\quad+\frac{1}{L^{D}} \sum_{\mathbf{p}_{1}, \ldots, \mathbf{p}_{N-1}} \frac{g_{L}(Q) g_{L^{\prime}}(Q)}{g_{L}(\sqrt{m E}) g_{L^{\prime}}(\sqrt{m E})} \frac{Y_{[L]}^{*}(\hat{\mathbf{Q}}) Y_{\left[L^{\prime}\right]}(\hat{\mathbf{Q}})}{m E-\mathbf{Q}^{2}} .
\end{aligned}
$$

Using the relation given in Eq. (A25), $\mathcal{D}_{[L],\left[L^{\prime}\right]}$ is linked to a generalized Lüscher formula in $D$-dimensional space,

$$
\begin{aligned}
& \frac{2}{\pi} \frac{(2 \pi)^{D}}{(m E)^{\frac{D-2}{2}}} i^{L-L^{\prime}} \mathcal{D}_{[L],\left[L^{\prime}\right]}(\sqrt{m E}) \\
& =\delta_{[L],\left[L^{\prime}\right]} \cot \delta_{L}^{(D)}(\sqrt{m E})-\mathcal{M}_{[L],\left[L^{\prime}\right]}(\sqrt{m E}),
\end{aligned}
$$

where $\mathcal{M}_{[L],\left[L^{\prime}\right]}$ is a generalized Lüscher zeta function in $D$ dimensional space,

$$
\begin{aligned}
& \frac{\pi}{2} \frac{(m E)^{\frac{D-2}{2}}}{(2 \pi)^{D}} \mathcal{M}_{[L],\left[L^{\prime}\right]}(\sqrt{m E})=i \delta_{[L],\left[L^{\prime}\right]} \frac{\pi}{2} \frac{(m E)^{\frac{D-2}{2}}}{(2 \pi)^{D}} \\
& \quad-\frac{1}{L^{D}} \sum_{\mathbf{p}_{1}, \ldots, \mathbf{p}_{N-1}} \frac{i^{L-L^{\prime}} g_{L}(Q) g_{L^{\prime}}(Q)}{g_{L}(\sqrt{m E}) g_{L^{\prime}}(\sqrt{m E})} \frac{Y_{[L]}^{*}(\hat{\mathbf{Q}}) Y_{\left[L^{\prime}\right]}(\hat{\mathbf{Q}})}{m E-\mathbf{Q}^{2}} \\
& \quad+\delta_{[L],\left[L^{\prime}\right]} \int \frac{Q^{D-1} d Q}{(2 \pi)^{D}}\left(\frac{g_{L}^{(D)}(Q)}{g_{L}^{(D)}(\sqrt{m E})}\right)^{2} \frac{1}{m E-\mathbf{Q}^{2}} .
\end{aligned}
$$

Therefore, the inverse of $\tau_{[L],\left[L^{\prime}\right]}^{(D)}$ is explicitly related to the Lüscher formula by

$$
\begin{aligned}
& \frac{2}{\pi} \frac{(2 \pi)^{D}}{(m E)^{\frac{D-2}{2}}}\left[\frac{g_{L}(\sqrt{m E}) g_{L^{\prime}}(\sqrt{m E})}{g_{L}(K) g_{L^{\prime}}\left(K^{\prime}\right)} \tau^{(D)}\left(K, K^{\prime}\right)\right]_{[L],\left[L^{\prime}\right]}^{-1} \\
& =i^{L^{\prime}-L}\left[\delta_{[L],\left[L^{\prime}\right]} \cot \delta_{L}^{(D)}(\sqrt{m E})-\mathcal{M}_{[L],\left[L^{\prime}\right]}(\sqrt{m E})\right] .
\end{aligned}
$$

The generalized Lüscher zeta function can also be derived by considering the hyperspherical harmonic basis function expansion of the Green's function. In infinite volume, the hyperspherical harmonic basis expansion of the Green's function is given by

$$
\begin{aligned}
& \int \frac{d \mathbf{Q}}{(2 \pi)^{D}} \frac{e^{i \mathbf{Q} \cdot\left(\xi-\xi^{\prime}\right)}}{m E-\mathbf{Q}^{2}} \stackrel{\xi>\xi^{\prime}}{=}-i(m E)^{\frac{D-2}{2}} \\
& \quad \times \sum_{[L]} Y_{[L]}\left(\Omega_{\xi}\right) \mathcal{H}_{L}^{(1)}(\sqrt{m E} \xi) \mathcal{J}_{L}\left(\sqrt{m E} \xi^{\prime}\right) Y_{[L]}^{*}\left(\Omega_{\xi^{\prime}}\right) .
\end{aligned}
$$

Similarly to the expansion of an infinite volume Green's function, the expansion of a finite volume Green's function may be written as

$$
\begin{aligned}
& \frac{1}{L^{D}} \sum_{\mathbf{p}_{1}, \ldots, \mathbf{p}_{N-1}} \frac{e^{i \mathbf{Q} \cdot\left(\boldsymbol{\xi}-\xi^{\prime}\right)}}{m E-\mathbf{Q}^{2}} \stackrel{\xi>\xi^{\prime}}{=}(m E)^{\frac{D-2}{2}} \sum_{[L],\left[L^{\prime}\right]} Y_{[L]}\left(\Omega_{\xi}\right) \\
& \quad \times\left[\delta_{[L],\left[L^{\prime}\right]} \mathcal{N}_{L}(\sqrt{m E} \xi)-\mathcal{M}_{[L],\left[L^{\prime}\right]}(\sqrt{m E}) \mathcal{J}_{L}(\sqrt{m E} \xi)\right] \\
& \quad \times \mathcal{J}_{L^{\prime}}\left(\sqrt{m E} \xi^{\prime}\right) Y_{\left[L^{\prime}\right]}^{*}\left(\Omega_{\xi^{\prime}}\right) .
\end{aligned}
$$

Combining Eqs. (A38) and (A39), we obtain

$$
\begin{aligned}
& \frac{1}{L^{D}} \sum_{\mathbf{p}_{1}, \ldots, \mathbf{p}_{N-1}} \frac{e^{i \mathbf{Q} \cdot\left(\boldsymbol{\xi}-\xi^{\prime}\right)}}{m E-\mathbf{Q}^{2}}-\int \frac{d \mathbf{Q}}{(2 \pi)^{D}} \frac{e^{i \mathbf{Q} \cdot\left(\boldsymbol{\xi}-\xi^{\prime}\right)}}{m E-\mathbf{Q}^{2}} \\
& \stackrel{\xi_{>} \xi^{\prime}}{=}(m E)^{\frac{D-2}{2}} \sum_{[L],\left[L^{\prime}\right]} Y_{[L]}\left(\Omega_{\xi}\right) \mathcal{J}_{L}(\sqrt{m E} \xi) \\
& \quad \times\left[i \delta_{[L],\left[L^{\prime}\right]}-\mathcal{M}_{[L],\left[L^{\prime}\right]}(\sqrt{m E})\right] \mathcal{J}_{L^{\prime}}\left(\sqrt{m E} \xi^{\prime}\right) Y_{\left[L^{\prime}\right]}^{*}\left(\Omega_{\xi^{\prime}}\right) .
\end{aligned}
$$

Next, using the plane wave expansion formula given in Eq. (A13) and also replacing $g_{L}^{(D)}(k)$ by $k^{L}$, we thus find, again, Eq. (A36), which may also suggest that the form factor $g_{L}^{(D)}$ may be chosen as $g_{L}^{(D)}(k) \sim k^{L}$.
[1] J. Kambor, C. Wiesendanger, and D. Wyler, Nucl. Phys. B465, 215 (1996).

[2] A. V. Anisovich and H. Leutwyler, Phys. Lett. B 375, 335 (1996).

[3] S. P. Schneider, B. Kubis, and C. Ditsche, J. High Energy Phys. 02 (2011) 028.

[4] K. Kampf, M. Knecht, J. Novotny, and M. Zdrahal, Phys. Rev. D 84, 114015 (2011).
[5] P. Guo, I. V. Danilkin, D. Schott, C. Fernández-Ramírez, V. Mathieu, and A. P. Szczepaniak, Phys. Rev. D 92, 054016 (2015).

[6] P. Guo, I. V. Danilkin, C. Fernández-Ramírez, V. Mathieu, and A. P. Szczepaniak, Phys. Lett. B 771, 497 (2017).

[7] G. Colangelo, S. Lanz, H. Leutwyler, and E. Passemar, Phys. Rev. Lett. 118, 022001 (2017).

[8] V. Efimov, Phys. Lett. 33B, 563 (1970). 
[9] E. Braaten and H. W. Hammer, Phys. Rep. 428, 259 (2006).

[10] M. V. Zhukov, B. V. Danilin, D. V. Fedorov, J. M. Bang, I. J. Thompson, and J. S. Vaagen, Phys. Rep. 231, 151 (1993).

[11] H. W. Hammer, C. Ji, and D. R. Phillips, J. Phys. G 44, 103002 (2017).

[12] M. Lüscher, Nucl. Phys. B354, 531 (1991).

[13] K. Rummukainen and S. A. Gottlieb, Nucl. Phys. B450, 397 (1995).

[14] N. H. Christ, C. Kim, and T. Yamazaki, Phys. Rev. D 72, 114506 (2005).

[15] V. Bernard, M. Lage, U.-G. Meißner, and A. Rusetsky, J. High Energy Phys. 08 (2008) 024.

[16] S. He, X. Feng, and C. Liu, J. High Energy Phys. 07 (2005) 011.

[17] M. Lage, U.-G. Meißner, and A. Rusetsky, Phys. Lett. B 681, 439 (2009).

[18] M. Döring, U.-G. Meißner, E. Oset, and A. Rusetsky, Eur. Phys. J. A 47, 139 (2011).

[19] R. A. Briceño and Z. Davoudi, Phys. Rev. D 88, 094507 (2013).

[20] M. T. Hansen and S. R. Sharpe, Phys. Rev. D 86, 016007 (2012).

[21] P. Guo, J. Dudek, R. Edwards, and A. P. Szczepaniak, Phys. Rev. D 88, 014501 (2013).

[22] P. Guo, Phys. Rev. D 88, 014507 (2013).

[23] S. Kreuzer and H. W. Hammer, Phys. Lett. B 673, 260 (2009).

[24] S. Kreuzer and H. W. Hammer, Eur. Phys. J. A 43, 229 (2010).

[25] S. Kreuzer and H. W. Grießhammer, Eur. Phys. J. A 48, 93 (2012).

[26] K. Polejaeva and A. Rusetsky, Eur. Phys. J. A 48, 67 (2012).

[27] R. A. Briceño and Z. Davoudi, Phys. Rev. D 87, 094507 (2013).

[28] M. T. Hansen and S. R. Sharpe, Phys. Rev. D 90, 116003 (2014).

[29] M. T. Hansen and S. R. Sharpe, Phys. Rev. D 92, 114509 (2015).

[30] M. T. Hansen and S. R. Sharpe, Phys. Rev. D 93, 096006 (2016); 96, 039901(E) (2017).
[31] R. A. Briceño, M. T. Hansen, and S. R. Sharpe, Phys. Rev. D 95, 074510 (2017).

[32] H.-W. Hammer, J.-Y. Pang, and A. Rusetsky, J. High Energy Phys. 09 (2017) 109.

[33] H. W. Hammer, J. Y. Pang, and A. Rusetsky, J. High Energy Phys. 10 (2017) 115.

[34] U.-G. Meißner, G. Ríos, and A. Rusetsky, Phys. Rev. Lett. 114, 091602 (2015); 117, 069902(E) (2016).

[35] M. Mai and M. Döring, Eur. Phys. J. A 53, 240 (2017).

[36] M. Mai and M. Döring, Phys. Rev. Lett. 122, 062503 (2019).

[37] M. Döring, H. W. Hammer, M. Mai, J. Y. Pang, A. Rusetsky, and J. Wu, Phys. Rev. D 97, 114508 (2018).

[38] F. Romero-López, A. Rusetsky, and C. Urbach, Eur. Phys. J. C 78, 846 (2018).

[39] P. Guo, Phys. Rev. D 95, 054508 (2017).

[40] P. Guo and V. Gasparian, Phys. Lett. B 774, 441 (2017).

[41] P. Guo and V. Gasparian, Phys. Rev. D 97, 014504 (2018).

[42] P. Guo and T. Morris, Phys. Rev. D 99, 014501 (2019).

[43] T. D. Blanton, F. Romero-López, and S. R. Sharpe, J. High Energy Phys. 03 (2019) 106.

[44] F. Romero-López, S. R. Sharpe, T. D. Blanton, R. A. Briceño, and M. T. Hansen, J. High Energy Phys. 10 (2019) 007.

[45] T. D. Blanton, F. Romero-López, and S. R. Sharpe, Phys. Rev. Lett. 124, 032001 (2020).

[46] M. Mai, M. Döring, C. Culver, and A. Alexandru, Phys. Rev. D 101, 054510 (2020).

[47] P. Guo, M. Döring, and A. P. Szczepaniak, Phys. Rev. D 98 , 094502 (2018).

[48] P. Guo, Phys. Lett. B 804, 135370 (2020).

[49] P. Guo and M. Döring, Phys. Rev. D 101, 034501 (2020).

[50] P. Guo, Phys. Rev. D 101, 054512 (2020).

[51] P. Guo and B. Long, Phys. Rev. D 101, 094510 (2020).

[52] P. Guo, arXiv:2007.04473.

[53] P. Guo and B. Long, arXiv:2007.10895.

[54] M. T. Hansen, F. Romero-López, and S. R. Sharpe, J. High Energy Phys. 07 (2020) 047.

[55] M. Fabre de la Ripelle, Ann. Phys. (N.Y.) 147, 281 (1983).

[56] M. Fabre de la Ripelle, Few-Body Syst. 14, 1 (1993).

[57] C. Lovelace, Phys. Rev. 135, B1225 (1964). 